\title{
PARYLENE SUPPORTED UNCOOLED THERMOELECTRIC INFRARED DETECTOR WITH UMBRELLA LIKE ABSORBER
}

\author{
M. J. Modarres-Zadeh", Z. S. Carpenter, and R. Abdolvand \\ Dynamic Microsystems Lab, Oklahoma State University, Tulsa, Oklahoma, USA
}

\begin{abstract}
In this work a very small $(20 \mu \mathrm{m} \times 20 \mu \mathrm{m})$ uncooled surfacemicromachined thermoelectric infrared detector is fabricated and tested. This TE detector features a P-doped polysilicon/Nichrome thermocouple embedded into a layer of Parylene-N to provide structural support. This sensor also features an umbrella-like IR absorber with the area of $\sim 19 \mu \mathrm{m} \times 19 \mu \mathrm{m}$ resulting in a $90 \%$ fill factor.
\end{abstract}

At room temperature, a responsivity of $\sim 150 \mathrm{~V} / \mathrm{W}$ with a time constant of $1.3 \mathrm{~ms}$ is measured from the fabricated devices in vacuum when viewing a $500 \mathrm{~K}$ blackbody without any concentrating optics. NEP and $\mathrm{D}^{*}$ are calculated to be $2.6 \mathrm{e}-10$ watt and $7.7 \mathrm{e} 6 \mathrm{~cm}^{*} \mathrm{~Hz}^{\wedge} 0.5 / \mathrm{w}$, respectively.

\section{INTRODUCTION}

Infrared (IR) or thermal imaging cameras have come a long way from their early days of development and MEMS technology has played an important role in the substantial progress made in the last few decades. Today, focal planar arrays (FPA) are fabricated with 100's of thousand detection cells integrated with CMOS interface circuit [1]. The applications of IR cameras have continuously grown with the technology and today it pertains to military, industrial, consumer electronics, and entertainment sectors. However, the relatively high cost of IR cameras is still a limiting factor in their availability into our everyday life.

IR detectors mainly drop into two categories: photon detectors and thermal detectors. Photon detectors have fast responses and they offer very good signal to noise ratio [1]. But, they require cryogenic cooling [1] which greatly increases the system cost/power consumption and limits the mobility of those systems. At room temperature, thermal noises terribly reduce the performance of these detectors. On the other hand, thermal detectors do not have this limitation and operate at room temperature. In these detectors, the incident radiation is absorbed causing a change in a temperature of an element. This change could be measured through several different mechanisms. Bolometer, pyroelectric, and thermoelectric (TE) detectors are examples of such class of IR detectors. In bolometers, a temperature sensitive resistor is used to measure the amount of the absorbed radiation. For these detectors, one limitation is that the instrument (substrate) temperature should be well stabilized [2]. In pyroelectric detectors, internal polarization happens due to temperature change in the pyroelectric material. To create the temperature change, the incoming radiation should be chopped. Adding a mechanical chopper is not favorable for portable and low-power applications. In thermoelectric detectors, Seebeck effect is used to measure the temperature difference between the absorber (hot junction) and the substrate (cold junction). These detectors have relatively low responsivity which makes the readout circuit complicated. However, there is no need for a mechanical chopper or tight temperature stabilization.

In this paper, the focus is to improve the sensitivity of the thermoelectric detectors by improving the thermal isolation resistance between the hot and the cold junctions. There have been many efforts dedicated to developing these detectors in the past $[3$, $4,5,6]$. This work continues the trend of decreasing the detector size, using folded thermal isolation arms, and introduc the application of a high thermally-resistive polymer (i.e. Parylene) to enhance thermal isolation and subsequently improve the responsivity. The physical design of the device and the materials used are presented as well as simulations of the transient thermal response. Lastly, the fabrication of the device is briefly discussed and the experimental results are presented.

\section{DESIGN}

As shown in Figure 1, the detector has an umbrella like absorber that covers most of the device area. This absorber is connected to a membrane through a post. This membrane is made of material with relatively high thermal conductivity and therefore the temperature of the absorber and the post is assumed to be fairly uniform. The TE hot junction is placed on this membrane. This membrane is suspended by two arms that are made of parylene and TE wires where parylene provides the required mechanical strength. On the silicon substrate a thin layer of silicon nitride is deposited to avoid low resistive path between the TE wires.

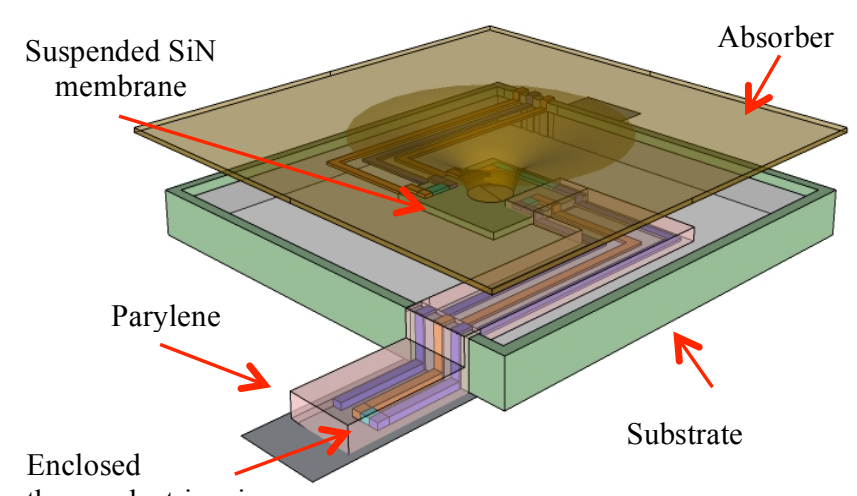

thermoelectric wires

Figure 1: The schematic view of the detector showing the suspended silicon nitride membrane in the center of the device in between the absorber and the substrate, and how the thermoelectric wires are embedded inside the parylene arms.

In a thermoelectric detector, TE wires are the most important components of the detector since they directly generate the output voltage and define the responsivity. To compare different TE materials, ZT $\left(=\frac{\sigma \cdot S^{2}}{k}\right.$ where $\mathrm{S}, \mathrm{k}$, and $\sigma$ are Seebeck coefficient, thermal conductivity and electrical conductivity, respectively) is a relevant figure of merit. For example at room temperature, bismuth telluride exhibits one of the highest ZT values (1.14) [7]. However, this material is not CMOS compliant. In this work polysilicon is chosen as one of the TE wires. ZT of silicon is low (0.01) [8] but it is readily available in the CMOS process which greatly reduces the fabrication cost and complexity. For the second TE wire, nichrome is chosen because of its low thermal conductivity [9] and chemical resistivity to hydrofluoric-based release processes.

To improve the device performance, the thermal resistance between the absorber and the substrate should be increased. This can be realized by looking at the DC responsivity equation [3]:

$$
\mathrm{R}=\mathrm{n} . \mathrm{N} . \mathrm{S} / \mathrm{G}
$$

Where $\mathrm{n}$ is the absorption efficiency, $\mathrm{N}$ is the number of thermocouples, $\mathrm{S}$ is the seebeck coefficient, and G is the thermal 
conduction ( $1 /$ thermal resistance) from the hot to cold junctions. Since the thermal conductivity of the TE wires is large, the first consideration is to reduce their cross sections and make them as small as possible. In this work, based on the detector size, the 20 $\mu \mathrm{m}$ long polysilicon wire has the thickness and width of $125 \mathrm{~nm}$ and $1 \mu \mathrm{m}$, respectively. This wire does not have the enough mechanical strength to hold the suspended absorber. Another material has to be added to provide structural support for the fragile wires. Silicon dioxide and silicon nitride have been used by others $[4,10]$. In this work, parylene- $\mathrm{N}$ is chosen because of its low thermal conductivity. Comparing the thermal conductivity of parylene $(\sim 0.1 \mathrm{~W} /(\mathrm{m} . \mathrm{K}))[11]$ to silicon dioxide $(>1 \mathrm{~W} /(\mathrm{m} . \mathrm{K})$ for thicknesses larger than $250 \mathrm{~nm}$ )[12] states that using parylene can greatly increase the thermal resistance between the hot and cold junctions. There are different types of parylene. Among them, parylene-C has the lowest thermal conductivity; however, its melting point is about $290{ }^{\circ} \mathrm{C}$ [11] which limits the processes that can be carried out on the wafer after the film deposition. Parylene$\mathrm{N}$ has slightly higher thermal conductivity $(125 \%)$, but its melting point is close to $420{ }^{\circ} \mathrm{C}$ [11]. Since the absorber is being deposited at high temperature after parylene deposition, parylene- $\mathrm{N}$ was preferred over the other types.

Another consideration in the design of the detector is choosing the number of the TE pairs. This can be determined by looking into the responsivity equation. The objective is to reduce the thermal conduction from the hot to cold junctions $(\mathrm{G})$ which consists of heat loss due to the TE wires, atmosphere, and the supporting parylene arms. By placing the detectors in vacuum, heat loss due to conduction through the gas can be negligible [13]. If the thermal conduction through the supporting arms was higher than the TE wires, increasing the number of the TE pairs could lead to increase in the responsivity. However, in this work, the dimension of the parylene $(2.5 \mu \mathrm{m}$ wide and $0.51 \mu \mathrm{m}$ thick $)$ is chosen in a way that the thermal conduction of the arms is much less than the TE wires. Based on the Fourier law of heat conduction, the product of thermal conductivity $(\mathrm{k})$ and cross section area (A) can be used to compare the heat losses. kAparylene $=1.6 \mathrm{e}-13$ and $\mathrm{kA}_{\mathrm{TE} \text { wires }}=5 \mathrm{e}-12$ are calculated assuming the thermal conductivity of about $40-50 \mathrm{~W} /(\mathrm{m} . \mathrm{K})[14]$ for polysilicon. These results show that the thermal loss due to the TE wires is 30 times than the parylene supporting arms. In this case, adding more thermocouple pairs, does not improve the detector responsivity, therefore, the detectors presented here are designed to contain only one TE pair.

To further improve the sensitivity of a detector, the incoming radiation should be efficiently absorbed by the absorber. There are different ways to achieve high absorption such as using metal black coatings $[15,16]$ such as gold-black or using optical cavities. Among the metal-black coatings, gold-black has relatively low density, low reflection, and high thermal conduction. However, these coatings are not mechanically stable [16]. It has been shown that high absorption can be achieved in metal / dielectric / metal cavities [17]. In this work, the cavity is formed on the absorber. Nichrome and silicon nitride are used as the metal layers and dielectric film, respectively. Since metals have large extinction coefficient, they exhibit high absorption but they also have high reflection due to their high refractive index which greatly reduces the absorption. To lower the refractive index, the metal layer can be made very thin [17]. According to [17]

$$
n=k=\left(\frac{\sigma}{2 * \omega * \varepsilon_{0}}\right)^{.5}
$$

(Where $\mathrm{k}$ is the extinction coefficient, $\sigma$ is electrical conductivity, $\omega$ is the incident light frequency in radians, and $\varepsilon_{0}=$ $8.85 * 1 \mathrm{e}-12$ is the vacuum permittivity), by reducing the metal layer thickness, its refractive index can be lowered. However, if the layer becomes too thin, the IR radiation might pass through without total absorption. The cavity is required so that any ray goes under multiple passes through the metal layer.

\section{SIMULATION}

Finite element analysis (COMSOL 4.1) is used to simulate the heat distribution and find the thermal response of the proposed detector. During the release step in the fabrication process (will be described later), it was observed that the parylene arms tend to bend upward due to the induced stress during the thermal cycles. As shown in Figure 2, two tethers are added to each side of the supporting arms to prevent the arms from deformation.

For the simulation, a constant power is applied to the absorber assuming $100 \%$ absorption and the heat distribution is depicted in Figure 2(a). To measure the transient response, the absorber temperature calculated from the previous part is set as the initial value for the absorber and the time it takes for the absorber to reach the same temperature as the substrate is plotted (Figure 2(b)). As it can be seen, the temperature fall time (or rise time) in the simulation is less than $8 \mathrm{~ms}$ with a time constant of $1.6 \mathrm{~ms}$.

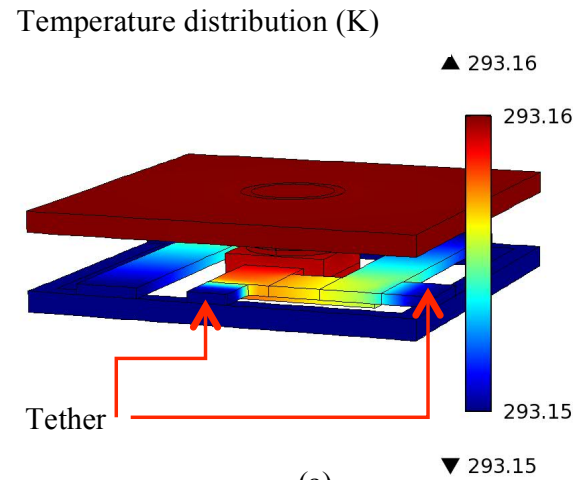

(a)

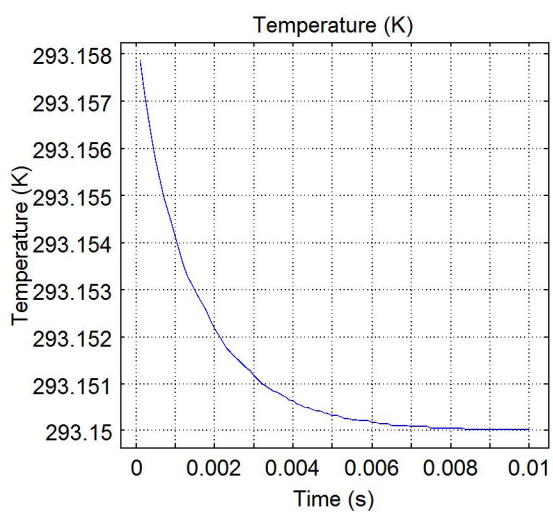

(b)

Figure 2: (a) Heat distribution when a constant power is applied to the absorber assuming $100 \%$ absorption (b) The temperature of the absorber versus time.

\section{FABRICATION}

Figure 3 represents the 7-step surface-micromachining process carried out for fabricating the thermoelectric detectors. The first step of the process is the deposition of a sacrificial PECVD $\mathrm{SiO}_{2}$ layer, which is patterned to serve as the platform for the suspended absorber. Next, LPCVD silicon nitride is deposited and patterned by dry etching to serve as the insulation layer for the electrical connections on top of both the substrate and the absorber post in the middle (Figure 3(a)). The TE junctions are configured with P- 
type polysilicon and nichrome. P-type polysilicon is deposited using LPCVD and then annealed at $950^{\circ} \mathrm{C}$ for $30 \mathrm{~min}$ to activate the dopants. Sheet resistance of $350 \mathrm{Ohm} /$ square was measured using the four point probe technique. Then nichrome is sputtered and patterned by lift-off. To improve the adhesion of nichrome to the substrate a thin layer of chromium is sputtered prior to that.

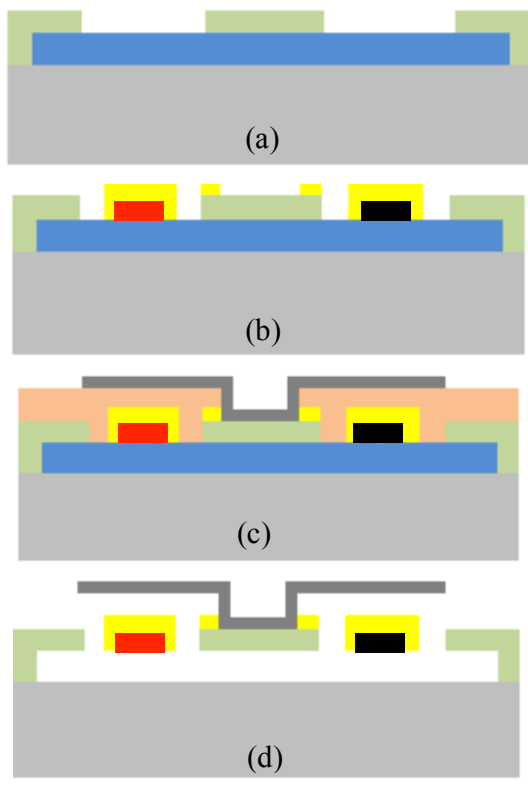

$$
\begin{aligned}
& \text { Silicon nitride Silicon Silicon dioxide } \\
& \text { - Thermoelectric material } 1 \text { Parylene } \\
& \text { - Thermoelectric material } 2 \text { Sacrificial layer } \\
& \text { - Absorber layer }
\end{aligned}
$$

Figure 3: The fabrication process flow, (a) sacrificial $\mathrm{SiO}_{2}$ layer deposition followed by silicon nitride deposition/patterning, (b) TE wire deposition/patterning followed by parylene deposition, (c) second sacrificial layer deposition followed by absorber formation, (d) Releasing the device by removing the sacrificial layers.

Then, supporting arms are formed from the parylene film deposited in a parylene-coating chamber (PDS 2010 system) at room temperature which is patterned in oxygen based plasma (Figure 3(b)). After fabricating the supporting arms, the second sacrificial layer is deposited which is $4 \mu \mathrm{m}$ of PECVD silicon dioxide and patterned to create access to the silicon nitride membrane (Figure 3(c)). Next step is the fabrication of the absorber which is comprised of three layers: (1) the bottom layer is sputtered nichrome with the sheet resistance of $40 \mathrm{Ohm} / \mathrm{square}$. (2) The second layer is $1 \mu \mathrm{m}$ of PECVD silicon nitride. (3) The very top layer is another sputtered nichrome layer with a sheet resistance of $>377 \mathrm{Ohm} / \mathrm{square}$. The absorber is then is anchored to the post through the patterned hole in the sacrificial layer as shown in Figure 3(c). To improve the adhesion of the absorber to its post, a thin layer of silicon nitride ( $300 \mathrm{~nm}$ thick) is deposited prior to the absorber deposition. The process of patterning the absorber is a combination of wet and dry etching: Silicon nitride layers are dry etched while the nichrome layers are wet etched in nichrome TFN. Lastly, both sacrificial layers are removed in an HF solution to completely release the structure (Figure 3(d)). The different layers along with their dimensions are summarized in Table 1 .
Table 1: Layers with their dimensions.

\begin{tabular}{|cccc|}
\hline $\begin{array}{c}\text { Layer } \\
\text { name }\end{array}$ & Thickness $(\mathrm{nm})$ & Width $(\mu \mathrm{m})$ & Length $(\boldsymbol{\mu m})$ \\
\hline Polysilicon & 125 & 1 & $\sim 20$ \\
\hline Nichrome & 250 & 2 & $\sim 20$ \\
\hline Parylene & 510 & 2.5 & $\sim 20$ \\
\hline membrane & 345 & 5 & 5 \\
\hline Absorber & 1000 & 19 & 19 \\
\hline
\end{tabular}

For the smallest fabricated device, the device area is $20 \mu \mathrm{m} \times 20$ $\mu \mathrm{m}$ and the absorber area is about $19 \mu \mathrm{m} * 19 \mu \mathrm{m}$ leading to a fill factor of as high as $90 \%$ as depicted in Figure 4. The same fabrication procedure is used to make arrays of $4 \times 4$ (Figure 5).

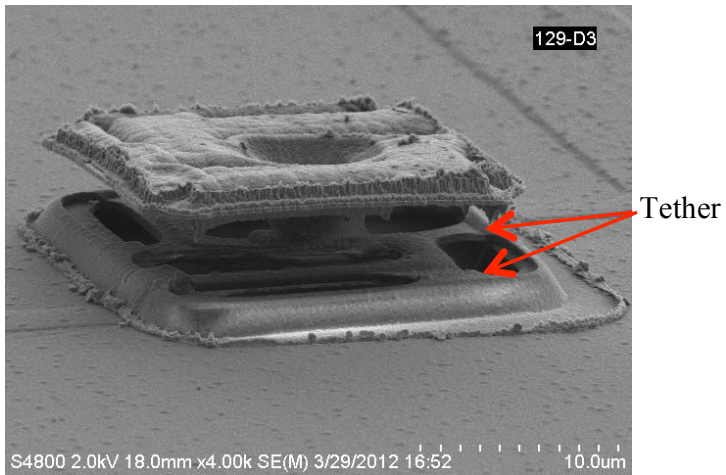

Figure 4: A fully fabricated device. There is an unwanted ring around the device created during the step of absorber patterning.

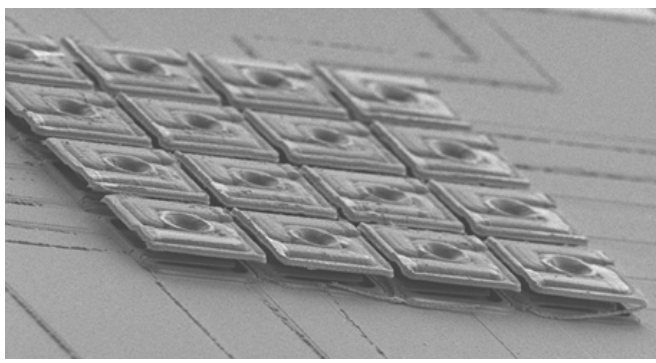

Figure 5: $4 \times 4$ array of detectors.

\section{RESULTS}

For measuring the responsivity, the detector is placed inside a vacuum probe station. On the top of the probing chamber, there is Zinc-Selenide window which let us probe the device by a CCD camera. After probing, a Ge window (3-12 $\mu \mathrm{m})$ is added to ensure blocking of the visible light.

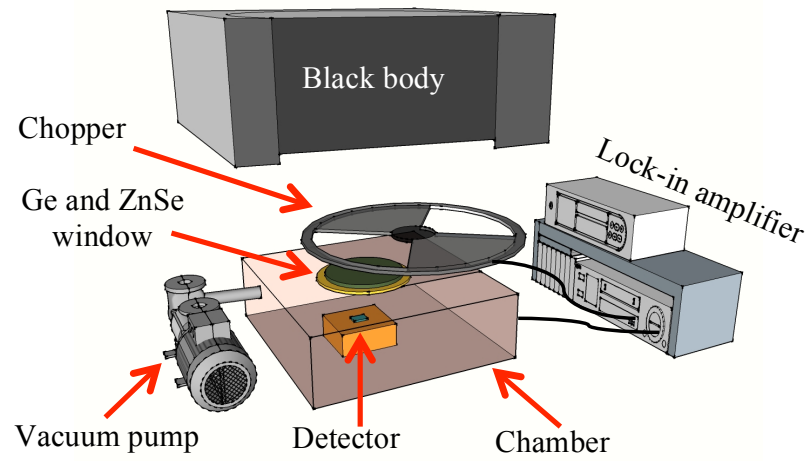

Figure 6: The measurement setup schematic, the vacuum probe station aperture is much smaller than the chopper blade opening. 
A $500 \mathrm{~K}$ black body is then placed in front of the chamber as an IR source. To suppress noise associated with DC measurement and gain a more accurate result, a chopper is placed between the chamber and the black body (as shown in Figure 6). A lock in amplifier is then used to measure the detector output signal. The amplified waveform is then averaged and shown in Figure 7. The responsivity of the device is calculated by dividing the device output voltage by the incoming IR radiation power. To measure the incoming IR power, a NIST calibrated sensor was replaced with the device and the power was measured under the same condition.

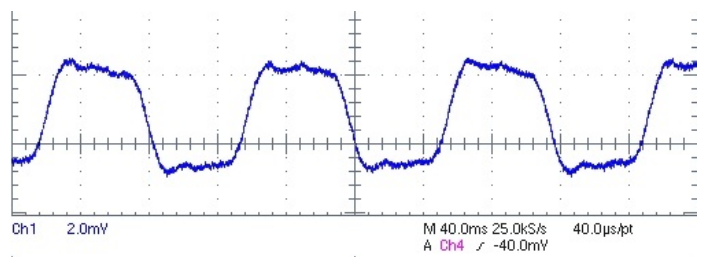

Figure 7: The device amplified and processed output signal.

The device responsivity versus frequency is plotted in Figure 8. It can be understood from the plot that thermal time constant of this detector is about $1.3 \mathrm{~ms}$ which is in agreement with the simulation.

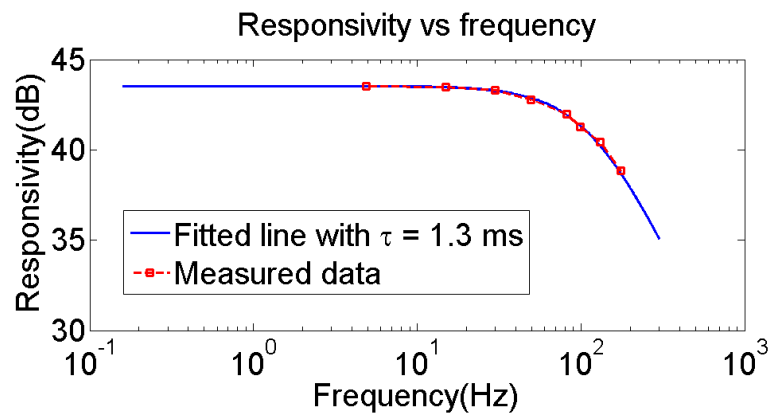

Figure 8: The device responsivity vs. frequency, the red dots are the measured data and the blue line is the fitted response with the time constant of $1.3 \mathrm{~ms}$.

If the devices are measured under open circuit condition, shot noise, flicker noise, and generation-recombination noise are zero and the only noise affecting the measurement is the thermal noise. As a result, the total noise, for the fabricated detector with resistances about $87 \mathrm{KOhm}$ is equal to Johnson noise which is calculated from $V n^{2}=4 K \cdot B . T \cdot R$ and is about $\sim 39 \mathrm{nV} /(\mathrm{Hz})^{\wedge} 0.5$. The $\mathrm{D}^{*}$ is calculated to be $7.7 \mathrm{e} 6 \mathrm{~cm}^{*} \mathrm{~Hz}^{\wedge} .5 / \mathrm{W}$.

\section{CONCLUSION}

Described was the design and fabrication of an uncooled thermoelectric IR detector that features one polysilicon-nichrome thermocouple enclosed in parylene arms. This detector is $20 \mu \mathrm{m} \mathrm{x}$ $20 \mu \mathrm{m}$ with an umbrella like absorber of $19 \mu \mathrm{m} \times 19 \mu \mathrm{m}$. A responsivity of $150 \mathrm{~V} / \mathrm{W}$ was measured in vacuum (1.5e-3 mbar) using a $500 \mathrm{~K}$ black body with a $(3-12 \mu \mathrm{m})$ Ge window. Finite element analysis was utilized to simulate the heat distribution and the transient response of the detector.

\section{ACKNOWLEDGMENT}

The work in this paper was supported by Amethyst research incorporated.

\section{REFERENCES}

[1] A. Rogalski, "Infrared detectors: status and trends", Progress in Quantum Electronics, Volume 27, Issues 2-3, 59-210(2003).

[2] M. C. Foote, "Temperature stabilization requirements for unchopped thermal detectors", Proc. of SPIE Conference on Infrared Technology and Applications XXV, 3698, 344-350(1999).

[3] P. W. Kruse, and D. D. Skatrud, Uncooled Infrared Imaging Arrays and Systems, Academic Presse, San Diego, 1997.

[4] M. C. Foote, E. W. Jones, and T. Caillat, "Uncooled thermopile infrared detector linear arrays with detectivity greater than $109 \mathrm{cmHz} 1 / 2 / \mathrm{W}$ ", IEEE Trans. Electron Devices 45, 18961902(1998).

[5] M. Hirota, Y. Ohta, and Y. Fukuyama, "Low-cost thermoelectric infrared FPAs and their automotive applications", Proc. SPIE 6940, (2008).

[6] D. Kryskowski, and J. Renken, "80 x 60 element thermoelectric infrared focal plane array for high-volume commercial use", Proc. SPIE 7298, (2009).

[7] D. Teweldebrhan, V. Goyal, and A. A. Balandin, "Exfoliation and Characterization of Bismuth Telluride Atomic Quintuples and Quasi-Two-Dimensional Crystals", Nano Letters, 12091218(2010).

[8] C. B.Vining, "Desperately Seeking Silicon", Nature, 451, 132(2008).

[9] P. Majumder, and A. Bhattacharyya, "A computational study of the impact of the Wiedemann-Franz-Lorenz law on the thermal response of nichrome cylinders", Modelling Simul. Mater. Sci. Eng., 16, 015006(2008).

[10] I. L. Choi, and K. D. Wise, "A Silicon-Thermopile-Based Infrared Sensing Array for Use in Automated Manufacturing", IEEE Trans. Electron Devices, 72-79(1986).

[11] N. Majid, S. Dabral, J. F. McDonald, "The ParyleneAluminum Multilayer Interconnection System for Wafer Scale Integration and Wafer Scale Hybrid Packaging", Electron. Mater. 18, 301(1989).

[12] T. Yamane, N. Nagai, S. Katayama, and M. Todoki, "Measurement of the thermal conductivity of silicon dioxide thin films using a 3-omega method", J. Appl. Phys.,91, 9772(2002).

[13] R. Goch, T. Schimert, W. McCardel, and B. Ritchey, "Waferlevel vacuum packaging for MEMS", J. Vacuum Sci. Technol. A, 17, 2295-2299(1999).

[14] A. D. McConnell, U. Srinivasan, M. Asheghi, and K. E. Goodson, "Thermal Conductivity of Doped Polysilicon Layers", J. Microelectromech. Syst. 10, 360 (2001).

[15] M. Hirota, Y. Nakajima, M. Saito, and M. Uchiyama, " $120 * 90$ element thermoelectric infrared focal plane array with precisely patterned Au-black absorber," Sens. Actuators A, Phys., 135, 146(2007).

[16] J. Lehman, E. Theocharous, G. Eppeldauer, and C. Pannell, "Gold-black coatings for freestanding pyroelectric detectors", Meas. Sci. Technol., 14, 916(2003).

[17] P. A. Silberg, "Infrared absorption of three layer films", J. Opt. Soc. Amer., 47, 575-578(1957).

\section{CONTACT}

*M. J. Modarres-Zadeh, tel: +1-918-850-8381;

jeff.modarres_zadeh@okstate.com 